\title{
Progress in the development of the neutron flux monitoring system of the French GEN-IV SFR: simulations and experimental validations.
}

\author{
C. Jammes, P. Filliatre, Zs. Elter, V. Verma, G. de Izarra, H. Hamrita, M. Bakkali, N. Chapoutier, A-C. Scholer, \\ D. Verrier, C. Hellesen, S. Jacobsson Svärd, B. Cantonnet, J-C. Nappé, P. Molinié, P. Dessante, R. Hanna, \\ M. Kirkpatrick, E. Odic, F. Jadot
}

\begin{abstract}
The neutron flux monitoring system of the French GEN-IV sodium-cooled fast reactor will rely on hightemperature fission chambers installed in the reactor vessel and capable of operating over a wide-range neutron flux. The definition of such a system is presented and the technological solutions are justified with the use of simulation and experimental results.
\end{abstract}

\section{INTRODUCTION}

$\mathrm{F}^{\mathrm{R}}$ RANCE has a long experience of about 50 years in designing, building and operating sodium-cooled fast reactors (SFR) such as RAPSODIE, PHENIX and SUPER PHENIX. Fast reactors feature the double capability of both reducing nuclear waste and saving nuclear energy resources by burning actinides. Since this reactor type is one of those selected by the Generation IV International Forum, the French government asked, in the year 2006, CEA, namely the French Alternative Energies and Atomic Energy Commission, to lead the development of an innovative GEN-IV nuclear-fission power demonstrator. The major objective is to improve the safety and availability of an SFR.

In this paper, we show that the architecture of the neutron flux monitoring (NFM) system will rely on in-vessel hightemperature fission chambers (HTFC) featuring wide-range

C. Jammes, P. Filliatre, G. de Izarra are with CEA, DEN, DER, Instrumentation, Sensors and Dosimetry Laboratory, Cadarache, F-13108 Saint-Paul-lez-Durance, France (telephone: +33 44257 4714, e-mail: christian.jammes@cea.fr)

Zs. Elter is with Chalmers University of Technology, Department of Applied Physics, Division of Nuclear Engineering, SE-412 96 Göteborg, Sweden

V. Verma, C. Hellesen, S. Jacobsson Svärd are with Division of Applied Nuclear Physics, Uppsala University, Box 516, SE-75120 Uppsala, Sweden

H. Hamrita, M. Bakkali are with CEA, DRT, LIST, Sensors and Electronic Architecture Laboratory, Saclay, F-91191 Gif Sur Yvette, France

N. Chapoutier, A-C. Scholer, D. Verrier are with AREVA NP, 10 rue Juliette Récamier F-69456 Lyon, France

B. Cantonnet, J-C. Nappé are with PHONIS France S.A.S, Nuclear Instrumentation, Avenue Roger Roncier, B.P. 520, F-19106 Brive Cedex, France

P. Molinié, P. Dessante, R. Hanna, M. Kirkpatrick, E. Odic are with Supéléc, Department of Power and Energy System, F-91192 Gif Sur Yvette, France

F. Jadot, CEA, DEN, DER, ASTRID Project Group, Cadarache, F-13108 Saint-Paul-lez-Durance, France flux monitoring capability based on the higher order Campbelling method. The definition of such a system is presented and the technological solutions are justified with the use of simulation and experimental results.

\section{SYSTEM DEFINITION AND DESIGN}

\section{A. Application of systems engineering methodology}

The NFM system is one of the important measurement systems in order to insure a high level of both nuclear safety and power plant availability.

One recalls that the main objective of nuclear safety is the achievement of proper operating conditions, prevention of accidents and mitigation of accident consequences, resulting in protection of workers, the public and the environment from undue radiation hazards [1].

In systems engineering, the availability of a system is its ability to be ready to achieve its required mission in defined operational conditions, assuming that required resources and existing support means are present [2]. In order to comply with this key operational requirement, the NFM system has to be both reliable and maintainable, that is capable of achieving a continuous mission and undergoing modifications and repairs, respectively. A system with these capabilities is said to feature dependability. In addition, the nuclear safety of a nuclear reactor is actually another dependability requirement, which is called innocuity in systems engineering [2].

The definition and design of the NFM of the French GENIV SFR is being conducted in accordance to the methodology of systems engineering. This is an iterative process that is composed of three main activities [2]:

- Definition of technical requirements: analysis and translation of end-user expectations into consistent and exhaustive technical requirements.

- Definition of logical architecture: definition of the elements constituting the functional, behavioral, temporal views of the system (functions, input-output flows, modes, transitions, triggers, etc.), and of their arrangement.

- Definition of physical architecture: definition of the elements constituting the physical view or architecture of the system (system elements/components, physical interfaces, 
connectors, etc.), and of their arrangement fitting the logical architecture.

\section{B. Main functional requirements}

The main functions of the NFM system are both reactivity control and power level monitoring from startup to full power. It is also required to monitor possible changes in neutron flux distribution within the core region in order to prevent any local melting accident.

\section{Requirements for integration}

The neutron detectors will have to be installed inside the reactor vessel because locations outside the vessel will suffer from severe disadvantages; (i) The installation of neutron detectors under the vessel is not feasible due to the presence of a core-catcher that prevents from inserting neutron guides in this region; (ii) The lateral and upper neutron shields, the role of which is to limit the damage and radiological impact of the neutrons outside the core, will also dramatically decrease the neutron flux amplitude, making thus difficult any neutron detection outside the vessel itself above or by the sides of the vessel.

Another important point is to limit the number of in-vessel detectors in order to impact the mechanical structures as low as possible, and to alleviate their installation and maintenance.

At the present stage of the French GEN-IV SFR project, two regions for the HTFC in-vessel installation are considered. The first region is the one above the core that is referred as the above-core-structure (ACS) region. The main ACS components are the absorber rod drive mechanisms and the core outlet temperature sensors. A few HTFC installation locations are thus available. These neutron detectors would be located at the bottom of the ACS, a few tens of centimeters above the fuel assembly heads. A limitation of this location is that the ACS moves with the rotating plugs during fuel handling operation so that HTFC signals would not be practical to manage during such operation. The second region is the one within the core support structure (CSS). The main advantages of the CSS region are the following; (i) CSS is a fixed structure and thus the HFTCs could be permanently serviceable even during fuel handling operation; (ii) There is no neutron absorbing material along the neutrons path, and; (iii) The sodium temperature is lower and stable. However, a limitation of this location comes from the difficulty to install HTFC and their appropriate guide-tubes.

\section{NEUTRON TRANSPORT SIMULATIONS}

Neutron-transport calculations allow us to select the two most appropriate regions for HTFC installation. Regions of interest are located inside the vessel, out of the core, beyond the neutron shield.

\section{A. Neutron transport modelling}

To calculate particle propagation over a long distance with many decades of attenuation, a Monte Carlo code such as MCNP [3] or TRIPOLI-4 [4] is needed. These neutron transport codes can be used in two modes: criticality mode, external source mode. Although MCNP has previously been used for neutronics studies in support of HTFC in-vessel location [5], TRIPOLI-4 is strongly recommended since its validation is under the responsibility of CEA itself. In the following paper, only the TRIPLOI-4 calculation route is presented.

Prior to any TRIPOLI-4 calculation, a geometry including the detector locations has to be generated. For this purpose, we used ROOT data analysis and visualization program [6] together with classes of templates written in the PYTHON programming language. This approach uses iterative structures for building the geometry, and it is modular when it comes to dealing with complex geometry.

As shown in [7] and [8], the results from the criticality calculation present large statistical uncertainty for a given calculation time. The large size of the core and the fact that the fission chambers are situated behind the neutron shield results in a rather low fraction of neutron histories reaching the detectors. For an improved quality of results, we see that a calculation scheme consisting of only criticality mode is not sufficient for core monitoring purposes. There is a need for variance reduction techniques for improved acceleration and reduced calculation times. However, a criticality calculation allows us to analyze detection rates at various locations and give the general trends for monitoring indications, which can be useful in qualifying a certain region as a possible detector location at the initial design phase of a neutron monitoring system.

In order to find a more reliable and efficient calculation route for core monitoring purposes without compromising on the computational efficiency, the use of the external source mode in TRIPOLI-4 is required. Fission sources are obtained from the ERANOS code package, and source definitions to be used in TRIPOLI-4 are then generated. One performs the neutron transport to the detector location, employing variance reduction techniques to provide appropriate biasing to the neutrons generated inside the core to be transported to faraway detector locations. Fig. 1 shows the calculation route used here. The results obtained show that we gain hugely on the statistical uncertainty using this approach as compared to criticality calculations.

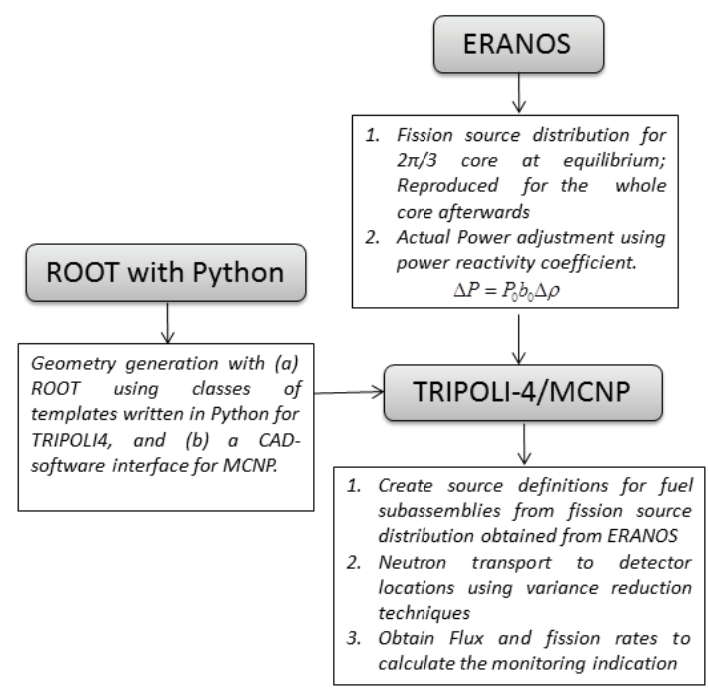

Fig. 1. Calculation route for Neutron transport Modelling. 


\section{B. In-vessel regions of HTFC installations}

The neutronics studies for the HTFC installations in ACS and CSS regions (Sect. II.C) were performed under the assumption that the reactor operate at full power. The results are summarized as follows:

1) The detector responses results in the ACS location confirm that this location is a good candidate for HTFC installation. The power operation and even the local change in the flux distribution due to an inadvertent control rod withdrawal (IRW) could be monitored and detected [5] [7] [8]. As an example, Fig. 2 displays the deviation of the fission chambers responses at the bottom of the ACS induced by the withdrawal of a peripheral control rod.

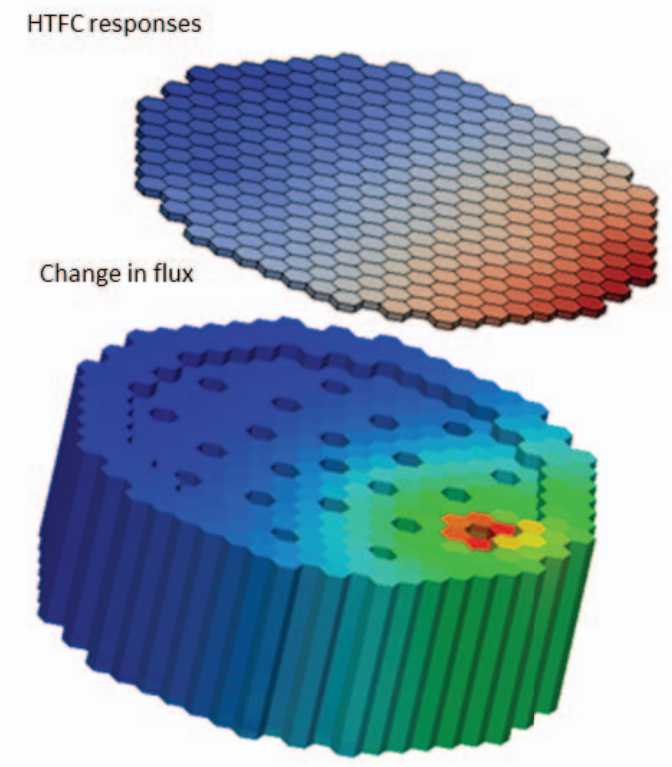

Fig. 2. Fission chamber responses at the bottom of the above-core structure (ACS). The detector responses reflect the change in the neutron flux within the core region due to an inadvertent control rod withdrawal (IRW). The fuel assemblies in red surround the control rod. They are the most sensitive to the change in flux.

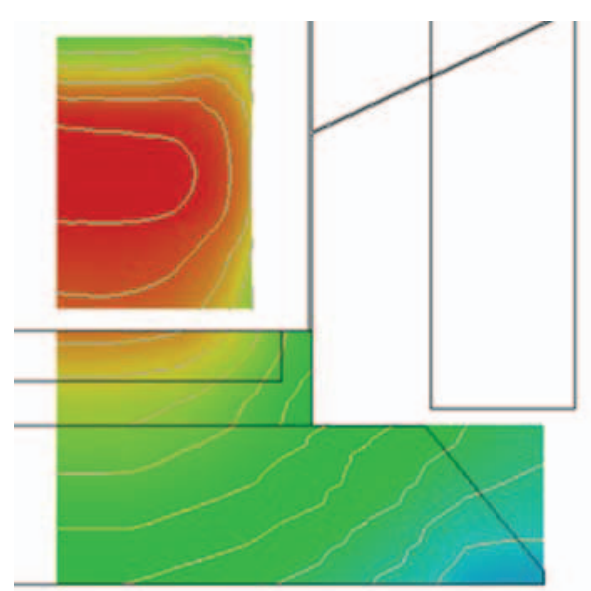

Fig. 3. Neutron importance map of the core. The highest importance is represented by the color red. The bottom part of the map is the core support structure (CSS).
2) The detector responses results for the CSS locations show that the neutron power and reactivity can be monitored with HTFCs installed in this region. However, when studying the control rod withdrawal scenario, the HTFC response deviations in possible positions are much lower in case of complete withdrawal. Fig. 3, which shows the fission neutron importance, helps us understand the reason why this detection performance is low. It clearly comes out that the fission neutrons are barely transported from the core to the CSS. As a result, this region is not satisfactory for detecting IRW, which predominantly causes a change in the neutron flux in the upper part of the core.

\section{HTFC DEVELOPMENT}

In this section, we present our endeavor to enhance the HTFC reliability through a comprehensive modeling of the detector itself, a more robust physical design and the study of the mineral insulation behavior at high temperature greater than $400^{\circ} \mathrm{C}$.

\section{A. HTFC modeling}

For many years, we have carried out a long run effort in conducting theoretical, modelling and experimental studies to improve the design and the signal analysis of fission chambers in order to improve their overall performance and dependability (Sect. II.A). This work is beneficial in defining HTFC with respect to their technical requirements, and in preparing experimental tests for their validation and qualification as items important to nuclear safety.

Constraints due to thermal expansion have been numerically shown to be negligible. However, the decrease of the resistivity of the insulators with temperature and irradiation can be an issue as far as partial discharges are concerned [9].

The development of tools based on the suite DARWIN [10] makes it possible to assess, for a given HTFC and its location within the vessel, the expected fission rate, its evolution with irradiation time, its sensitivity to energetic domains of interest (e.g. fast neutrons) [11], [12]. It has been shown that U235 is the isotope of choice to deliver a suitable fission rate for signal processing, and stable enough with time, provided the HTFC is not placed within the core or at reflector level [9]. It was also shown [13] that the surface mass of the coating has to be below $1 \mathrm{mg} / \mathrm{cm}^{2}$ or so to avoid significant self-absorption, a constraint that leads to large diameter $(48 \mathrm{~mm})$, multielectrode designs. Finally, the activation of the coating and the structures of the HTFC can be computed to anticipate on postuse handling.

The saturation curves are computed with tools derived from the equations of [14]. An experimental validation is presently undertaken at MINERVE reactor of CEA that would put some constraints on physical parameters involved in the so-called recombination regime [15]. Fig. 4 shows saturation curves at various pressures and fission rates. 
A comprehensive tool [16], [17] has been developed that simulates the ionization of the gas by the fission products, the transportation of the charges, the induced current. Observable quantities such as the mean pulse, the charge spectrum, the mean current (hence the sensitivity in current mode) the spectral density (hence the sensitivity in Campbelling mode), can be computed, their dependence to the design parameters (geometrical specifications, gas composition, bias voltage) can be studied. For instance, increasing the gas mass crossed by the fission product (either by increasing the gas pressure or increasing the gap between the electrodes) increases the pulse height, as long as the fission product is not stopped in the gas itself, but also increases the pulse length. Using gas mixtures with molecular gases to shorten the pulse length is not advised in SFR, as the molecules would dissociate under the gamma radiation. This tool has received a partial experimental validation [18]. Also, it is possible to estimate the fraction of the signal due to gamma interactions with the HTFC structures in current or Campbelling mode [19]. It is thus possible, for a given region in the reactor, to choose a set of design parameters that is a good compromise for monitoring the flux over ten orders of magnitude up to nominal power.

(a) Current

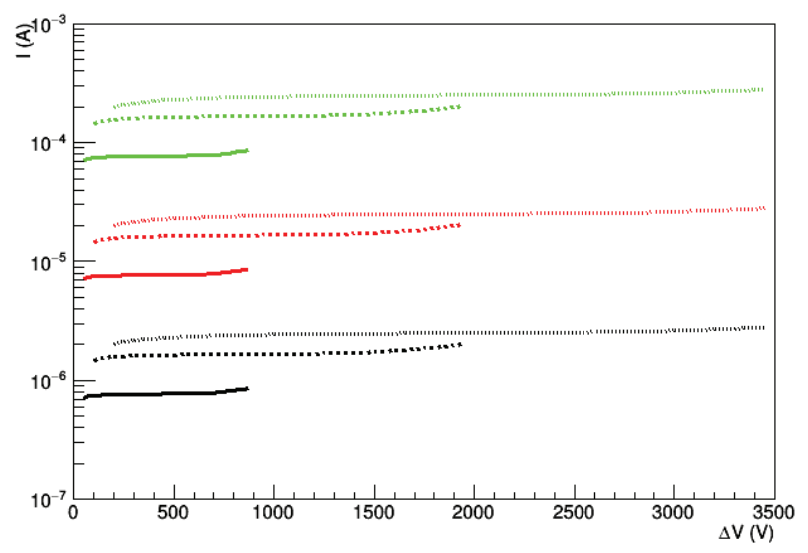

(b) Normalized current

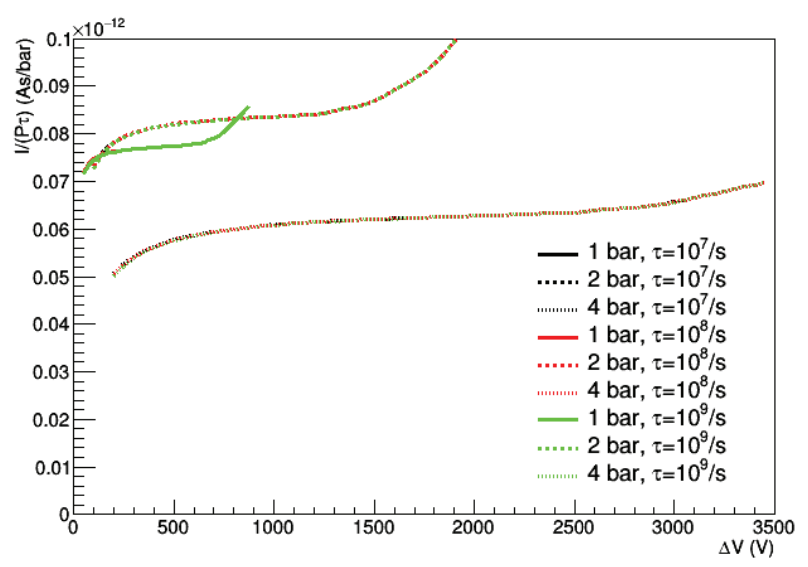

Fig. 4 HTFC saturation curves for various argon pressure and fission rates of the fissile layer. The electrode spacing is $1.5 \mathrm{~mm}$. The fission chamber signal is expressed in both (a) current and (b) current normalized to fission rate and pressure. Note that as long space charges are not taken into account, there is a perfect scaling in fission rate.

\section{B. Physical design}

During the last decades of the 20th century, CEA and Photonis jointly developed high temperature fission chambers for the PHENIX and SUPER PHENIX fast reactor [20] [21]. Two types of detectors were hence developed:

- CFUC06 for Phénix, which was filled with pure argon.

- CFUC07 for Superphénix, which was filled with $\mathrm{Ar}+4 \% \mathrm{~N} 2$ gas mixture, in order to get larger flux dynamic.

The physical architectures of CFUC06 and CFUC07 were based on a guard ring structure (Fig. 5), which allows us to increase the insulation between High Voltage (HV) and Signal electrodes, especially at high temperature of about $550^{\circ} \mathrm{C}$. This is due to the fact that no ceramic insulator is placed between the two electrodes, and that two cables are used, one for $\mathrm{HV}$ and one for Signal, with no connection of the electrodes to the ground reference.

This experience benefited to Photonis' know-how of the HTFC manufacturing process. More specially, the experience feedback shows that alumina exhibits satisfactory insulating properties, and after testing different metal materials that Inconel 600 allows reliable fission chambers. Our improvements in modelling (Sect. IV.A) and analyzing the fission chamber signal (see further in Sect. IV.D and V) make it possible to change the internal structure of HTFC to a onecable structure (Fig. 5): in this configuration one alone cable is used for both HV and Signal, and the cathode is connected to the ground. Thus, this change allows simplifying the design of HFTC by decreasing the quantity of piece parts and making it more robust by increasing the piece part size. Photonis is currently manufacturing prototypes that must be tested with CEA at high temperature.

(a) Guard-ring structure

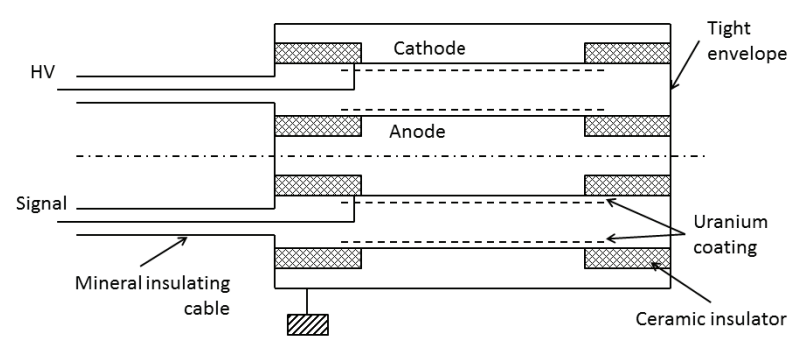

(b) One-cable structure

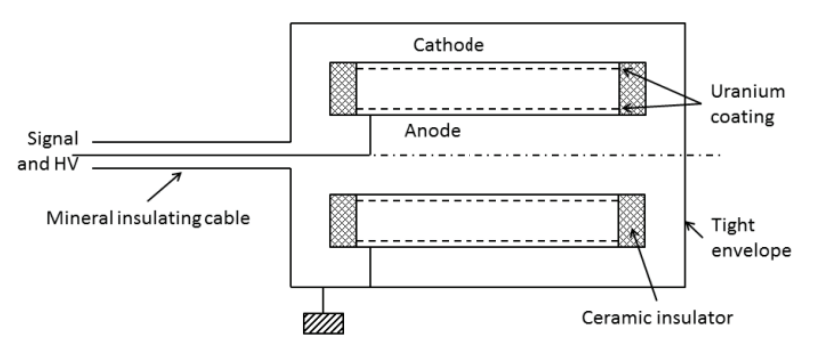

Fig. 5. Sketch of (a) guard ring and (b) a one-cable structure of a fission chamber from an electric point of view. 


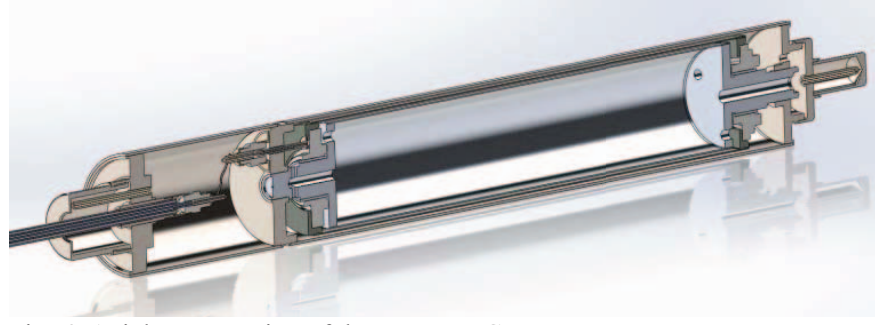

Fig. 6. Axial cross section of the new HTFC structure.

Fig. 6 shows the new structure of this HTFC, which is designed to take into account the difference between each material thermal expansion coefficient in the three dimensions. The main characteristics of these prototypes are:

- Electrodes and detector body in Inconel 600.

- Three electrodes, two cathodes and one anode, the detector envelope being one cathode.

- An outer diameter of $48 \mathrm{~mm}$.

- A gap between the electrode of 1 or $1.5 \mathrm{~mm}$.

- A sensitive length of $210 \mathrm{~mm}$.

- Pure argon filling gas, to avoid nitrogen disappearing at high temperature.

- A uranium thickness of $1 \mathrm{mg} / \mathrm{cm}^{2}$.

- The same high immunity parasitic and mineral insulated cable as the one qualified for CFUC06 and CFUC07, manufactured by Thermocoax Company.

\section{Impact of partial discharges}

The HTFC fissile layer is subject to partial discharges at high temperature when the electric field between the electrodes is greater than $200 \mathrm{~V} / \mathrm{mm}$. These discharges give rise to pulses similar to the desired neutron pulses generated by the fission chamber itself, which may bias the HTFC count rate at start-up only.

The exact mechanism leading to these electrical discharges is unclear. Gas pressure on the order of several bar and gap distances on the order of mm means that these discharges are occurring under conditions significantly inferior to the Paschen limit. Extrapolation of the Paschen curve for Argon to high pressure suggests that discharges should not be observed for electric fields below a value of about $10 \mathrm{kV} / \mathrm{mm}$.

Current work is focused on modeling and experimentation to identify the location in the HTFC where the discharges may occur. Initial calculations showed that the maximum field attained in a faultless HTFC should be well below the $10 \mathrm{kV} / \mathrm{mm}$ threshold, and so consideration has been given to triple points and possible defaults in the fissile layer or HTFC structure where electrical field enhancement may occur.

Fig. 7 shows the results of a calculation of the electric field for a default consisting of the detachment of the insulator from the outer conductor. This calculation gave a value of electric field at about $7 \mathrm{kV} / \mathrm{mm}$, while other (perhaps less probable) types of default involving triple points combined with insulation defaults resulted in values as high as $20 \mathrm{kV} / \mathrm{mm}$. (a) Axisymmetric cross section

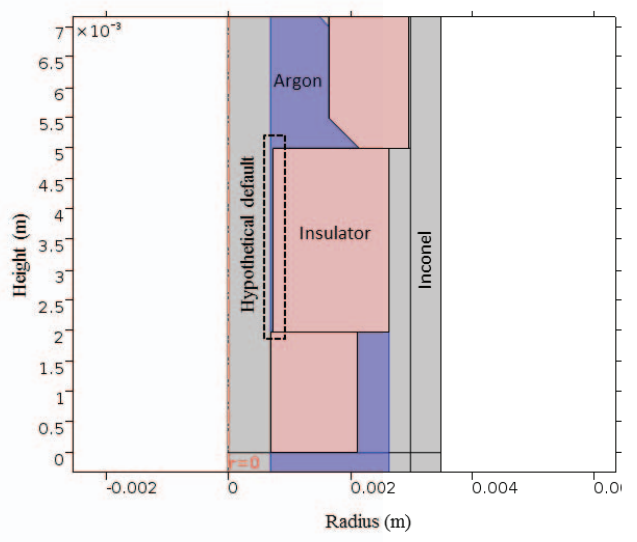

(b) Electric field norm (MV/m)

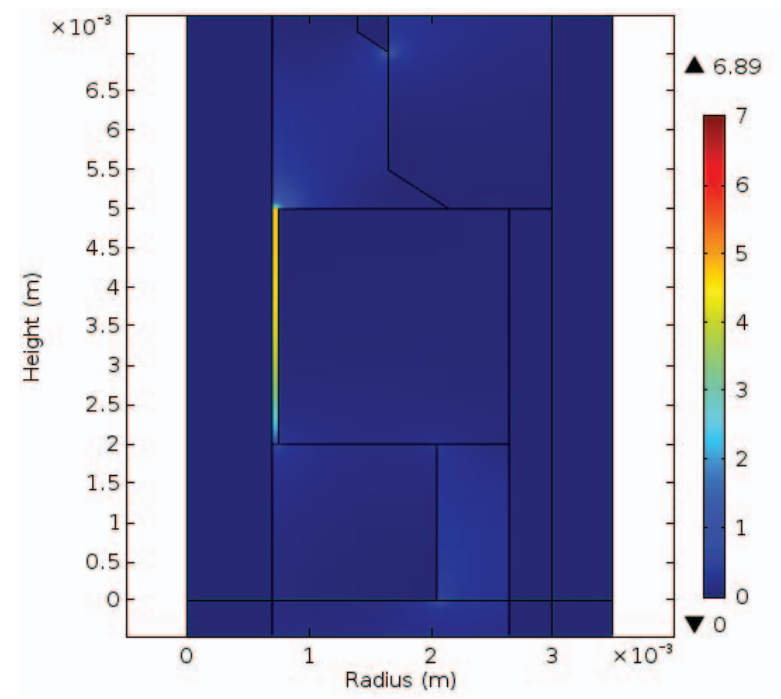

Fig. 7. Calculation of the enhanced electric field created by a hypothetical default of insulator detachment from HTFC outer conductor. Maximum resulting field of about $7 \mathrm{kV} / \mathrm{mm}$ or $7 \mathrm{MV} / \mathrm{m}$.

In addition, the effect of temperature seems to be critical for the formation of these discharges, as they are not observed at low temperature. Future effort shall be made to determine how the higher temperature may either lead to defaults or cause other conditions to arise which are responsible for the discharges. Ideally the goal is to eliminate these discharges by the change of design after understanding the exact mechanism of their creation.

\section{Experimental discrimination of partial discharges}

If this proves not to be practical, we have also worked on a signal analysis method able to discriminate the desired neutron signal from the signal created by the discharges. As displayed in Fig. 8, we have experimentally verified the feasibility of this method. The histogram showed in this figure is the result of analysis of $10^{4}$ neutron pulses and as many pulses due to partial discharges. Fig. 8 gives the distribution of the width (at 


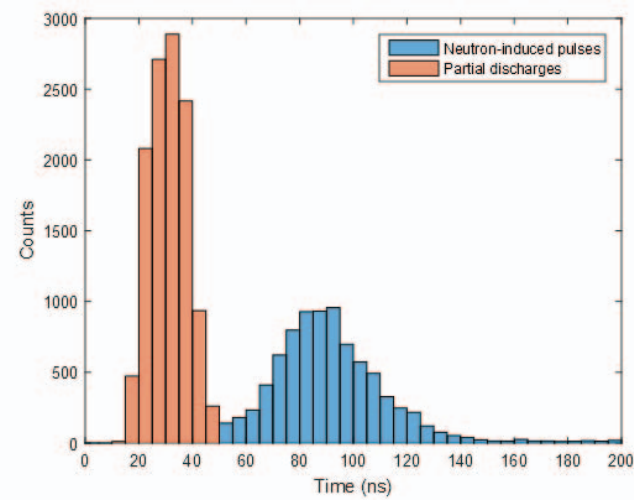

Fig. 8. Experimental discrimination of neutron pulses from partial discharges.

the half maximum) of the collected pulses. The distribution has two well separated peaks. The peak related to the longer width can be identified with the neutron pulses (since the results match the date provided by Photonis). It was assumed that pulses with shorter width are created by the partial discharges. This was verified through measurements without neutron flux.

The partial discharges distribution shows that the mean half-height width is about $30 \mathrm{~ns}$. We have verified this latest distribution with the same chamber but without neutron flux.

Despite the apparent similarity between the two types of pulses, we have shown that we are able to discriminate these partial discharge pulses of those produced by the fission products in the HTFC using the half-height width of the measured pulses.

\section{V.SIGNAL PROCESSING DEVELOPMENT}

The fission chambers traditionally operate in three different modes (namely the pulse, the Campbelling and the current mode for low, middle-high and high count rates respectively). These operational modes require different electronic systems hence resulting a deficiency of robustness. Recently it was shown that the overlap of these modes is not necessarily guaranteed. For the newly developed NFM a unified mode is proposed via the higher order Campbelling methods.

\section{A. High-order Cambelling method}

The fission chamber signal is described by a filtered Poisson process. One recalls that the Campbelling mode of such signal is based on the signal variance, which is the second-order cumulant as well. The generalization of this method gives proportionality between the higher order cumulants of the signal and its count rate $s_{0}$ :

$$
\kappa_{n}^{(s t)}=s_{0}\left\langle x^{n}\right\rangle \int_{-\infty}^{+\infty} f(t)^{n} d t
$$

where the proportionality is given by a coefficient including the raw moments of the amplitude distribution $w(x)$ and the integral of the pulse shape $f(t)$. Hence the application of Higher order Campbelling methods essentially means the measurement of the higher order cumulants $\kappa_{n}$. [22], [23].

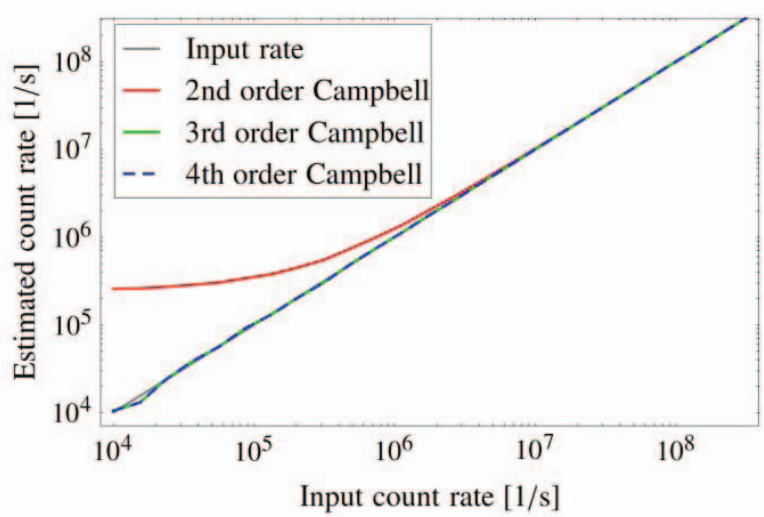

Fig. 9. Noise rejection and linearity of HOC methods (in presence of Gaussian white noise)

It was shown through numerical simulations that the application of the third order Campbelling mode is reliable and can guarantee linear estimation over a wide count rate. It can also sufficiently reject the impact of electronic noise and suppress the impact of pulses not originating from neutron events (Fig. 9).

Due to the relatively fast convergence of the third order cumulant estimation the monitoring of transient events with HOC modes is also reliable.

Campbelling mode based on the third-order cumulant will permit to ensure the HTFC response linearity over the entire neutron flux range using a signal processing technique that is simple enough to satisfy design constraints on electric devices important for nuclear safety.

\section{B. Experimental calibration}

An experimental campaign devoted to the required calibration process of HOC method was also carried out at the MINERVE facility. The calibration implies the determination of the proportionality coefficient, therefore the determination of the pulse shape and the pulse amplitude distribution in Eq. (1).

Our experimental setup consisted of a current-sensitive CFUL01 fission chamber placed in the reflector zone of the MINERVE reactor. The CFUL01 is a fair representation of the HTFC. The fission chamber was connected to a wide range pre-amplifier. Then, the output signal was digitized at high sampling frequency during a large time span with an advanced digital oscilloscope.

At low reactor power levels pulses were collected separately. Even at low power there is a small possibility to measure pileup events, hence during the post processing of the results the pileup events were found and these events were not taken into account during the determination of the calibration coefficient. These results provided information about the amplitude distribution of the pulses hence the raw moments of this distribution. Based on the collected pulses the mean pulse shape was also defined. This information enabled us to determine the calibration coefficients summarized in Table 1.

At medium and high power levels longer signals were collected. At medium levels the pulses moderately overlap. 
TABLE I. FISSION CHAMBER CALIBRATION COEFFICIENTS

\begin{tabular}{cl} 
CUMULANT ORDER & CALIBRATION COEFFICIENT \\
\hline 2 & $9.74 \cdot 10^{-19}$ \\
3 & $5.40 \cdot 10^{-24}$ \\
4 & $3.76 \cdot 10^{-29}$ \\
\hline
\end{tabular}

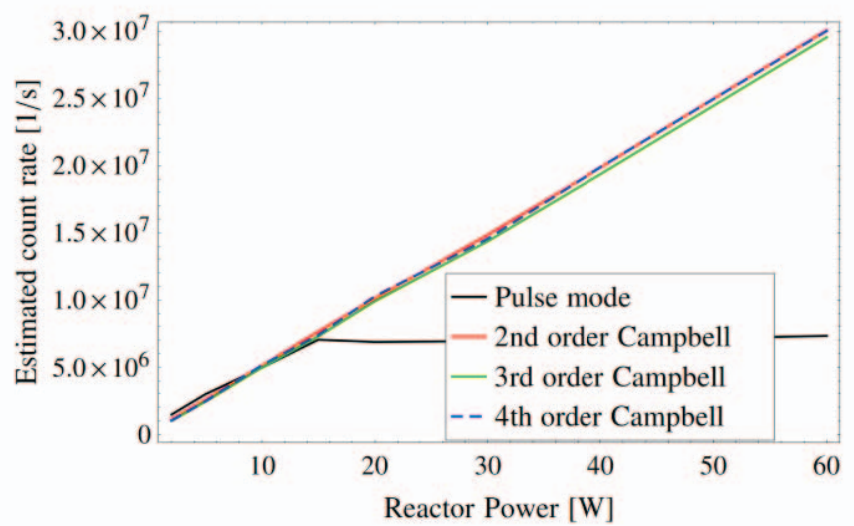

Fig. 10. Experimental validation of $\mathrm{HOC}$ technique linearity.

As a result, counting them separately is still possible to estimate the count rate of the signal. This provides an opportunity to estimate the count rate with simple pulse mode and compare with the estimation coming from the calibrated HOC mode.

As one can see in Fig. 10, the Campbelling results show a good agreement with the simple pulse counting estimation at low count rates. It is also shown that the HOC technique provides a linear estimation of the count rates at higher power levels as well. Hence the application of the HOC methods is reliable.

\section{Electronic implementation}

The electronic system design must meet a variety of requirements such as temperature stability, electromagnetic compatibility, response time, neutron flux estimation accuracy.

The electronic system contains an analog part and a digital part. The analog part is essentially made by the low noise fast current neutron flux preamplifier which is a key element in the electronic system. The low noise fast current neutron flux preamplifier converts the current signal from the HTFC to voltage signal with an input impedance of $50 \mathrm{ohms}$ to match the fission chamber cable. The input matching impedance enables a long connection (150 to $300 \mathrm{~m}$ ) between the detector and its associated electronic system. The detector can therefore be exposed to hard radiations conditions without damage for the electronics.

Then, an analog-to-digital converter digitalizes the voltage signal which is injected into a FPGA (Field Programmable Gate Array) circuit. Fig. 11 shows the functional architecture of the HOC method to be implemented in a FPGA. Here, the

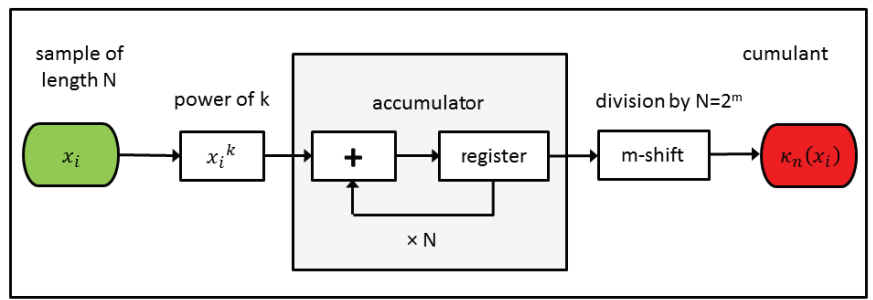

For $\mathrm{k}=2$ and 3

Fig. 11.Functional flow-block diagram of HOC method to be implemented in a FPGA circuit. The digitized input signal of length $\mathrm{N}$ is processed in order to estimate $\kappa_{2}$ and $\kappa_{3}$ cumulants. Each operation is executed within one clock cycle: exponentiation, summation and division. The summation is done with the use of an accumulator that consists of an adder and a register. The division by $\mathrm{N}=2^{\mathrm{m}}$ is carried out by a shift operator.

input signal is supposed to be centered, that is zero-meanvalued. The digitized input signal of length $\mathrm{N}$ is processed in order to estimate $\kappa_{2}$ and $\kappa_{3}$ cumulants. Given that the signal is centered, the processing is similar to computing two moments, which are actually two power series:

$$
\kappa_{n}=\frac{1}{N} \sum_{i=1}^{N} x_{i}^{k}, k=2 \text { or } 3
$$

\section{CONCLUSION AND PERSPECTIVES}

The development of the neutron flux measurement (NFM) system is still in progress. At present, the main features of the logical and physical architectures are defined. However, the dependability of the whole system is not matured enough. Its architecture will thus have to be enhanced to this purpose. Other important points to the NFM system are the neutron power calibration and the validation/qualification tests. The power calibration will be based on both a standard thermal balance and an innovative spectrometry monitoring of fluorine 20 that is an isotope produced by sodium activation. In order to perform the qualification program of the NFM system, we have recently initiated the preparation of functional and stress tests in the JOYO facility in Japan. These tests will be defined and performed in tight collaboration with Japanese Atomic Energy Agency (JAEA).

\section{ACKNOWLEDGMENT}

The study was performed in the framework of an on-going collaboration on the instrumentation and safety of sodium cooled fast reactors between Chalmers, Uppsala University and CEA with support from the Swedish Research Council. The authors are also grateful to AREVA for their contribution to this work and support within the TECNA project.

\section{REFERENCES}

[1] IAEA, IAEA Safety Glossary - Terminology used in nuclear safety and radiation protection, 2007th ed., IAEA, Ed. Vienna, Austria, 2007.

[2] A. Faisandier, "Systems Architecture and Design - Engineering and Architecting Multidisciplinary Systems Volume 3," Sinergy'Com, 2013.

[3] X-5 Monte Carlo Team, "MCNP - A General Monte Carlo N-Particle Transport, Version 5," LANL, Los Alamlos, NM, USA, LA-UR-03- 
$1987,2003$.

[4] C. M Diop et al., "TRIPOLI-4: a 3D continuous-energy Monte Carlo transport code," in PHYTRA1: First International Conference on Physics and Technology of Reactors and Applications, Marrakech, Morocco, 2007.

[5] C. Jammes et al., "Neutron flux monitoring system of the French GENIV SFR: Assessment of diverse solutions for in-vessel detector installation," Nucl. Eng. and Design, vol. 270, p. 273, 2014.

[6] R. Brun and F. Rademakers, "ROOT, an object-oriented data analysis framework," Nucl. Instrum. and Meth. A, vol. 389, p. 81, 1997.

[7] V. Verma, C. Hellesen, and C. Jammes, "Detectability of U-235 fission chambers in case of inadvertent control rod withdrawal in a French sodium-cooled fast reactor," in International Youth Nuclear Congress (IYNC2014), Burgos, Spain, 2014.

[8] V. Verma, C. Jammes, C. Hellesen, P. Filliatre, and S. Jacobsson Svärd, "Monte Carlo criticality mode limitations for the study of the French GEN-IV SFR neutron flux monitoring," Submitted to Ann. Nucl. Energy.

[9] P. Filliatre, C. C. Jammes, B. Geslot, and L. Buiron, "In vessel neutron instrumentation for Sodium-cooled fast reactors : type, lifetime and location," Ann. Nucl. Energ., vol. 37, p. 1435, 2010.

[10] A. Tsilanizara and C. M. Diop, "DARWIN: an evolution code system for a large range of applications," J. Nucl. Sci. Technol., vol. 37, p. 845, 2000.

[11] P. Filliatre, L. Oriol, C. Jammes, and L. Vermeeren, "Reasons why Plutonium 242 is the best fission chamber deposit to monitor the fast component of a high flux," Nucl. Instr. and Meth. A, vol. 593, p. 510 2008.

[12] P. Filliatre, L. Oriol, C. Jammes, and L. Vermeeren, "Joint estimation of the fast an thermal components of a high neutron flux with a two on-lin detector system," Nucl. Instr. and Meth. A, vol. 603, p. 415, 2009.

[13] C. Jammes, P. Filliatre, P. Loiseau, and B. Geslot, "On the impact of the fissile coating on the fission chamber signal," Nucl. Instr. and Meth. A, vol. 681, p. 101, 2012.

[14] S. Chabod, "Saturation current of miniaturized fission chambers," Nucl. Instr. and Meth. A, vol. 598, p. 578, 2009.

[15] P. Filliatre, B. Geslot, C. Jammes, and V. Lamirand, "Experimental study of columnar recombination in fission chamber," unpublished.

[16] P. Filliatre, C. Jammes, B. Geslot, and R. R. Veenhof, "A Monte Carlo simulation of the fission chambers neutron-induced pulse shape using the GARFIELD suite," Nucl. Instr. and Meth. A, vol. 678, p. 139, 2012.

[17] C. Jammes et al., "Research Activities in Fission Chamber Modeling in Support of the Nuclear Energy Industry," IEEE Trans. Nuc. Sci., vol. 57, p. $3678,2010$.

[18] B. Geslot et al., "New measurement system for on line in core highenergy neutron flux monitoring in materials testing reactor conditions," Rev. Sci. Instr., vol. 82, p. 033504, 2011.

[19] P. Filliatre, L. Vermeeren, C. Jammes, B. Geslot, and D. Fourmentel, "Estimating the gamma-ray contribution to the signal of fission chambers with Monte Carlo simulations, soumis à," Nucl. Instr. and Meth. A, vol. 648, p. 228, 2011.

[20] J-P. Trapp, S. Haan, L. Martin, J. Perrin, and M. Tixie, "igh temperature fission chambers: State-of-the-art," in Proc. Specialists' Meeting, InCore Instrumentation and Core Assessment, Mito-shi, Japan, 1996.

[21] C. Jammes, P. Filliatre, B. Geslot, T. Domenech, and S. Normand, "Assessment of the high temperature fission chamber technology for the French fast reactor program," IEEE Trans. Nucl. Sci., vol. 59, p. 1351, 2012.

[22] L. Pál, I. Pázsit, and Zs. Elter, "Comments on the stochastic characteristics of fission chamber signals," Nucl. Instrum. and Meth. A, vol. 763, p. 44, 2014.

[23] Zs. Elter, C. Jammes, I. Pázsit, L. Pál, and P. Filliatre, "Performance investigation of the pulse and Campbelling modes of a fission chamber using a Poisson pulse train simulation code," Nucl. Intrum. and Meth. A, vol. 774, p. 60, 2015.

[24] J. Leppänen, "Performance of woodcock delta-tracking in lattice physics applications using the SERPENT Monte Carlo reactor physics burnup calculation code," Ann. Nucl. Energy, vol. 37, p. 715, 2010.

[25] J. Leppänen, "Development of a new Monte Carlo reactor physics code," Helsinki University of Technology, Helsink, D.Sc. Thesis 2007.

[26] J. Ruggieri, J. Tommasi, and J. Lebrat, "ERANOS 2.1: International code system forGEN IV fast reactor analysis," in International Congress on Advances in Nuclear Power Plants (ICAPP 2006), Reno, NV, USA, 2006.

[27] F. Gauché, "Generation IV reactors and the ASTRID prototype: lessons from the Fukushima accident," Comptes Rendus Physique, vol. 13, p. $365,2012$.

[28] Python software foundation. Python. [Online]. https://www.python.org/ 